\title{
Local administration of Tiludronic Acid downregulates important mediators involved in periodontal tissue destruction in experimental periodontitis in
} rats

\author{
Flávia A.C. Furlaneto ${ }^{\mathrm{a}, *}$, Nara L.T. Nunes ${ }^{\mathrm{b}}$, Ricardo B. Souza ${ }^{\mathrm{c}}$, Kely O. Yamamoto ${ }^{\mathrm{b}}$, \\ Ivan Lima Oliveira Filho ${ }^{\mathrm{b}}$, Nicolly P.R. Frota ${ }^{\mathrm{a}}$, Hellíada Chaves ${ }^{\mathrm{b}}$, Mario Lisboa ${ }^{\mathrm{d}}$, Mario Taba Jr. ${ }^{\mathrm{a}}$, \\ Edilson Ervolino ${ }^{\mathrm{e}}$, Michel R. Messora ${ }^{\mathrm{a}}$ \\ ${ }^{a}$ Department of Surgery and Bucco-Maxillofacial Traumatology and Periodontology, Ribeirão Preto School of Dentistry, University of São Paulo - USP, Ribeirão Preto, SP, \\ Brazil \\ ${ }^{\mathbf{b}}$ Federal University of Ceará - UFC, Sobral, Ceará, Brazil \\ ${ }^{\mathrm{c}}$ Department of Biochemistry and Molecular Biology, Federal University of Ceará - UFC, Fortaleza, Ceará, Brazil \\ d Department of Morphology, Faculty of Medicine, Federal University of Ceará, Fortaleza, Ceará, Brazil \\ e Division of Histology, Department of Basic Sciences, Dental School of Araçatuba, São Paulo State University - UNESP, Brazil
}

\section{A R T I C L E I N F O}

\section{Keywords:}

Bisphosphonates

Periodontal disease

Bone

Inflammation

\begin{abstract}
A B S T R A C T
Objective: The purpose of this study was to evaluate whether local administration of TIL could influence the expression of the inflammatory mediators IL-1 $\beta$, TNF- $\alpha$, MMP-8 and COX-2 in rats with experimental periodontitis (EP).

Methods: Twenty-four adult male rats (Rattus norvegicus, albinus, Wistar) were assigned to groups C, EP, EP-TIL (C-Control group, EP-Periodontitis groups). On EP groups, a ligature was placed around maxillary 2nd molars on day 1. On group EP-TIL, $20 \mu \mathrm{L}$ of TIL solution $(1 \mathrm{mg} / \mathrm{kg}$ body weight) was injected into the subperiosteal palatal area adjacent to the maxillary 2nd molar every other day until euthanasia (day 11). Alveolar bone loss was morphometrically analyzed. mRNA expressions of IL-1 $\beta$, TNF- $\alpha$, MMP-8 and COX-2 were assessed by qPCR. IL-1 $\beta$, TNF- $\alpha$, MMP-8 and COX-2 were immunohistochemically analyzed. Data were analyzed statistically. Results: Group EP-TIL presented reduced alveolar bone loss when compared with group EP ( $p<0.05$ ). Group EP-TIL presented decreased mRNA expressions of IL-1 $\beta$, TNF- $\alpha$, MMP-8 and COX-2 and reduced immunolabeling of IL-1 $\beta$, TNF- $\alpha$ and MMP-8 when compared with group EP $(p<0.05)$. No differences regarding the immunolabeling of COX-2 were found when group EP-TIL was compared with the other groups $(p>0.05)$.

Conclusion: Within the limits of this study, it can be concluded that local administration of TIL downregulates important mediators involved in periodontal tissue destruction in ligature-induced periodontitis in rats.
\end{abstract}

\section{Introduction}

The host modulation of matrix metalloproteinase (MMP) or the blockage of inflammatory cytokines are approaches to block the progression of inflammatory bone loss observed in periodontitis (Kirkwood, Cirelli, Rogers, \& Giannobile, 2007). Therefore, inflammatory cell signaling pathways that generate these inflammatory and tissue destruction proteins have become promising therapeutic targets (Kirkwood et al., 2007). Bisphosphonates (BPs) are one of the categories of host-modulating agents that have been investigated in the periodontal therapy (Kirkwood et al., 2007).

BPs are synthetic chemical drugs that inhibit bone resorption by selective adsorption to mineral surfaces and subsequent internalisation by osteoclasts substantially affecting genesis and resorptive activity of these cells (Russell et al., 2007). BPs can also promote osteogenesis by stimulating osteoblastic differentiation (D'Aoust, McCulloch, Tenenbaum, \& Lekic, 2000). In fact, increases in osteoid matrix formation and in collagen synthesis were observed with the use of BPs (Guenther, Guenther, \& Fleisch, 1981; Reddy et al., 2005). Other effect attributed to a bisphosphonate (BP) was the reduction on bacterial

\footnotetext{
* Corresponding author at: Department of Oral \& Maxillofacial Surgery and Periodontology, Ribeirao Preto School of Dentistry, University of Sao Paulo - USP, Av. do Cafe, s/n 14040904, Ribeirao Preto, SP, Brazil.

E-mail addresses: flafurlaneto@usp.br (F.A.C. Furlaneto), naralhays@hotmail.com (N.L.T. Nunes), ricardobastosouza@gmail.com (R.B. Souza), kelyoy@hotmail.com (K.O. Yamamoto), ivan_oli2@hotmail.com (I.L. Oliveira Filho), nicollyfrota@yahoo.com.br (N.P.R. Frota), helliadachaves@yahoo.com.br (H. Chaves), marioplisboa@gmail.com (M. Lisboa), mtaba@forp.usp.br (M. Taba Jr.), eervolino@foa.unesp.br (E. Ervolino), m.messora@forp.usp.br (M.R. Messora).
} 
growth in experimental periodontitis in rats (Menezes et al., 2005). Some BPs also present anti-inflammatory effects by reducing the influx of neutrophils to the inflamed gingiva and/or the inhibition of pro-inflammatory mediators (Buduneli et al., 2007; Menezes et al., 2005). It has been reported that BPs may be an appropriate adjunctive treatment in the clinical management of periodontitis (Lane et al., 2005; Sharma \& Pradeep, 2012).

Tiludronic Acid (chloro-4-phenyl-thiomethylene-1,1-bisphosphonate) (TIL) is a non-nitrogen-containing BP mainly indicated for the treatment of Paget's disease of bone (Silverman, 2008). It inhibits osteoclast-mediated bone resorption (Rogers et al., 1999), disrupts cytoskeleton resorption of osteoclasts (Murakami et al., 1997), inhibits activity of these cells on the proton pump (David, Nguyen, Barbier, \& Baron, 1991) and leads to osteoclast apoptosis (Rogers et al., 1999). TIL also has anti-inflammatory effects, since it can dose-dependently restrain interleukin (IL)-6 production by osteoblasts (Tokuda, Kozawa, Harada, \& Uematsu, 1998) and the secretion of tumor necrosis factor (TNF)- $\alpha$, IL-1 $\beta$, IL- 6 and nitric oxide (NO) by activated macrophages (Mönkkönen, Simila, \& Rogers, 1998). It was demonstrated that TIL can inhibit MMP-1 and -3 , important enzymes for the degeneration of extracellular matrix constituents in periodontal diseases, in human periodontal ligament cells (Nakaya et al., 2000).

Recently, we demonstrated that local administrations of TIL solutions influenced osteoclasts, reduced alveolar bone resorption and the intensity of the local inflammatory response in experimental periodontitis (EP) in rats (Furlaneto et al., 2014). However, it is necessary to analyze how TIL influences periodontal tissues, examining the regulation of important inflammatory mediators involved in periodontal tissue destruction. The purpose of this study was to evaluate the influence of local administration of TIL on the genic expression and the immunolabeling of IL-1 $\beta$, TNF- $\alpha$, MMP-8 and cyclooxygenase (COX)-2 in rats with ligature-induced periodontitis.

\section{Materials and methods}

\subsection{Sample}

This study was conducted according to the ethical principles of animal experimentation, as well as standards for the didactic-scientific practice of vivisection and the Universal Declaration of Animal Rights by United Nations Educational, Scientific and Cultural Organization. The present research was performed after acceptance by the Ethics Committee on Animal Research at Federal University of Ceara-UFC, Fortaleza, CE, Brazil (protocol 028/2011).

A power calculation was executed to establish the sample size. The animal was considered the study unit. The sample size was calculated to provide $80 \%$ power to recognise significant differences among groups with a $95 \%$ confidence interval $(a=0.05)$, considering the means and standard deviations of the genic expression of IL- $1 \beta$ of the study by Lisboa et al. (2015). Thus, a sample size of eight animals per group was needed.

\subsection{Experimental model}

Twenty-four 3- to 4-month-old male Wistar rats (Rattus norvegicus, albinus, Wistar) weighing 250 to $300 \mathrm{~g}$ were used (Central Animal Facility of the UFC) in this study. The animals were maintained in a room with a 12-h light/dark cycle with temperatures from $22^{\circ} \mathrm{C}$ to $24^{\circ} \mathrm{C}$. All through the experiment, the rats were kept in plastic cages and fed with selected solid diet and water ad libitum. Before the study began, the rats were identified by a numeric code. According to a random numeric table generated by a computer software, the study coordinator (F.A.C.F.) allocated each rat into one of the following groups $(n=8)$ : 1) C (control): EP was not induced and TIL was not administered; 2) EP: EP was induced with a ligature and TIL was not administered; 3) EP-TIL: EP was induced with a ligature and TIL solutions at a dosage of $1 \mathrm{mg} / \mathrm{kg}$ body weight were locally administered. The allocation sequence was unknown to the investigators of the study (operators, outcome assessors and biostatistician).

\subsection{Induction of periodontitis with ligature}

Animals were anesthetized by an intramuscular injection of xylazine. $^{1}$ ( $6 \mathrm{mg} / \mathrm{kg}$ body weight) and ketamine ${ }^{2}(70 \mathrm{mg} / \mathrm{kg}$ body weight) on day 1 . After general anesthesia, each animal was placed on the operating table, which allowed keeping the rat's mouth open, facilitating access to posterior teeth of the maxillae. In groups EP and EP-TIL, a nylon ligature ${ }^{3}$ was placed around the cervical area of the maxillary second molars of each rat, remaining supragingivally at both buccal and lingual faces. The knots were positioned at the buccal face of the teeth. In group EP-TIL, $20-\mu \mathrm{L} \mathrm{TIL}{ }^{4}$ solutions ( $1 \mathrm{mg} / \mathrm{kg}$ body weight) were injected into the subperiosteal palatal area adjacent to the maxillary second molars on days 1, 3, 5, 7, and 9 in the morning. Throughout the experimental period, the animals were weighed every other day, and the doses of TIL were adapted accordingly (Furlaneto et al., 2014).

The animals were euthanized under anesthesia with a final solution of xylazine $(30 \mathrm{mg} / \mathrm{kg}$ body weight) and ketamine $(240 \mathrm{mg} / \mathrm{kg}$ body weight) 11 days after the placement of the ligatures. The hemimaxillae were excised and fixed in $4 \%$ formaldehyde for $24 \mathrm{~h}$ (Furlaneto et al., 2014). Samples of gingival tissues around maxillary second molars of each animal were collected and stored at $-80^{\circ} \mathrm{C}$.

\subsection{Morphometric analysis}

The hemimaxillae were chemically defleshed and stained with $1 \%$ aqueous methylene blue in order to enhance the visibility of the cementoenamel junction (CEJ). Standardized photographs of the buccal aspects of the hemimaxillae were obtained using a digital camera ${ }^{5}$ connected to a stereomicroscope ${ }^{6}$ and a computer, with an original magnification of $\mathrm{x} 16$. The images were evaluated with appropriated software. $^{7}$

The linear distances between alveolar bone crest and CEJ (ABC-CEJ $-\mathrm{mm}$ ) were measured in the interproximal area between the $1 \mathrm{st}$ and the 2nd molar, in the interproximal area between the 2nd and the 3rd molar and also at the long axes of the mesial and distal roots of the 2nd molar. The average of these linear measurements obtained from each animal was used to express the ABC-CEJ value, which represented the alveolar bone loss. Morphometric analysis was performed by a masked and calibrated examiner (N.P.R.F.).

\subsection{Quantitative reverse transcription-polymerase chain reaction ( $q P C R$ )}

The gingival samples were macerated under freezing with liquid nitrogen. Total RNA was extracted by guanidinium isothiocyanatephenol-chloroform method, using TRIzol ${ }^{\mathrm{TM} 8}$ and RNA was resuspended in diethyl pyrocarbonate (DEPC) ${ }^{8}$-treated water. RNA concentration was evaluated by spectrophotometry using the ratio of the absorbance at 260 and $280 \mathrm{~nm}$, and its integrity was analyzed by $1 \%$ agarose-gel electrophoresis with ethidium bromide $(10 \mathrm{mg} / \mathrm{mL})$. Complementary DNA (cDNA) was reverse transcribed from $5.0 \mu \mathrm{g}$ of total RNA by using an oligo (dT) primer ${ }^{8}$ and SuperScript III reverse transcript, ${ }^{8}$ according to manufacturer's instructions (Coura et al., 2015). The primers used are described in Table 1. Quantitative Real Time PCR (qPCR) for gene

\footnotetext{
${ }^{1}$ Rompam ${ }^{\star}$, Bayer Saúde Animal, São Paulo, SP, Brazil.

${ }^{2}$ Dopalen, Agribands, Paulínia, SP, Brazil.

${ }^{3}$ 3-0, Technofio, Goiânia, GO, Brazil.

${ }^{4}$ Tildren, Ceva Saúde Animal, Paulínia, SP, Brazil.

${ }^{5}$ Leica DFC 310 FX, Leica Microsystems CMS GmbH, Wetzlar, Germany.

${ }^{6}$ Leica MZ6, Leica Microsystems GmbH, Wetzlar, Germany.

${ }^{7}$ Image J - National Institutes of Health, Washington, DC, USA.

${ }^{8}$ Invitrogen, Life Technologies, Carlsbad, CA, USA.
} 
Table 1

Description of primer sequences.

\begin{tabular}{|c|c|c|c|c|}
\hline Description & Abbreviation & Sequence & Amplicon size & Access number/ Source \\
\hline Interleukin-1 $\beta$ & IL-1 $\beta$ & $\begin{array}{l}\text { R 5'- CCCTgCAgCTggAgAgTgTgg - } 3^{\prime} \\
\text { F 5'- TgTgCTCTgCTTgAgAggTgCT }-3^{\prime}\end{array}$ & 153 & NM_031512.2/NCBI \\
\hline Tumor necrosis factor- $\alpha$ & TNF- $\alpha$ & $\begin{array}{l}\text { F 5'- CggggTgATCggTCCCAACAA - } 3^{\prime} \\
\text { R 5'- gTggTTTgCTACgACgTgggC }-3^{\prime}\end{array}$ & 139 & NM_012675.3/NCBI \\
\hline Matrix metalloproteinase- 8 & MMP-8 & $\begin{array}{l}\text { F 5'- TggAgTgTgCCATCAACCCTgAC }-3^{\prime} \\
\text { R 5'- CACCATggTCTCTTgAgACgAAAgC }-3^{\prime}\end{array}$ & 88 & NM_022221.1/NCBI \\
\hline Cyclooxygenase-2 & $\mathrm{COX}-2$ & $\begin{array}{l}\text { F 5'- TCCAgTATCAgAACCgCATTgCCT - } 3^{\prime} \\
\text { R 5'- AgCAAgTCCgTgTTCAAggAggAT - } 3^{\prime}\end{array}$ & 149 & NM_017232.3/NCBI \\
\hline Glyceraldehyde-3-phosphate dehydrogenase & GAPDH & $\begin{array}{l}\text { F 5'-gggggCTCTCTgCTCCTCCC }-3^{\prime} \\
\text { R 5'CggCCAAATCCgTTCACCg }-3^{\prime}\end{array}$ & 108 & Coura et al., 2015 (20) \\
\hline
\end{tabular}

expression was performed using Mastercycler ${ }^{\curvearrowleft}$ ep realplex $4^{9}$ and Power SYBR Green Master Mix ${ }^{\circ 10}$ Each sample was analyzed in a total reaction volume of $20 \mu \mathrm{L}$, consisting of $0.1 \mu \mathrm{g}$ of cDNA, $10 \mu \mathrm{L}$ of $2 \mathrm{X}$ SYBR Green Master $\mathrm{Mix}^{\oplus 8}$ and $300 \mathrm{nM}$ of forward and reverse primers. ${ }^{8}$ Three replicates of each sample were amplified. The $2^{-\Delta \Delta \mathrm{Ct}}$ method (Livak \& Schmittgen, 2001) was used to determine the relative expressions of IL$1 \beta$, TNF- $\alpha$, MMP-8 and COX-2 mRNA. All values were expressed as mean \pm standard error mean (SEM) relative to the levels in group $C$ $(\mathrm{n}=8$ ), arbitrarily set to 1 (Coura et al., 2015). mRNA levels of the glyceraldehyde-3-phosphate dehydrogenase (GAPDH) gene was used as endogenous control (housekeeping gene) for the normalization of qPCR data.

\subsection{Immunohistochemical analyses}

The hemimaxillae were decalcified in 10\% EDTA solution. After complete decalcification, the specimens were processed and embedded in paraffin. Serial sections, $5 \mu \mathrm{m}$ thick, were obtained in a mesio-distal direction. Histologic sections representing the most central buccal-lingual portion in the area between first and second left maxillary molars were selected. Immunohistochemical processing was performed through the indirect immunoperoxidase method, as previously described (Furlaneto et al., 2014). The histologic slides containing samples from all the experimental groups were divided into four batches, and each batch was incubated with one of the following primary antibodies: rabbit anti-interleukin (IL)-1 $\beta,{ }^{11}$ goat anti-tumor necrosis factor (TNF)- $\alpha,{ }^{12}$ rabbit anti-matrix metalloproteinase (MMP)- $8^{13}$ or mouse anti- cyclooxygenase (COX)-2. ${ }^{14}$

Histologic sections were evaluated under light microscopy with an optical microscope ${ }^{15}$ by a masked and calibrated examiner (E.E.). The region analyzed comprised an area of $1000 \mu \mathrm{m}^{2}$ located at the center of the interdental alveolar septum between the maxillary first and second molars. The coronal limit of this area was the $\mathrm{ABC}$, from which the region analyzed extended apically for $1000 \mu \mathrm{m}$ (Furlaneto et al., 2014). The immunolabeling scores were determined as follows: score 0-no immunolabeling (total absence of immunoreactivity in the area); score 1-low immunolabeling pattern $(\cong 1 / 4$ of the area presenting immunoreactivity); score 2-moderate immunolabeling pattern ( $\cong 1 / 2$ of the area presenting immunoreactivity); score 3-high immunolabeling pat-

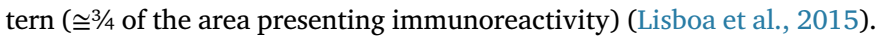

\subsection{Examiner Calibration}

All analyses were performed by masked examiners. To estimate the

\footnotetext{
${ }^{9}$ Eppendorf AG, Hamburg, Germany.

${ }^{10}$ Applied Biosystems, Foster City, CA, USA.

11 SC-7884, Santa Cruz Biotechnology, Santa Cruz, CA, USA

12 SC-1348, Santa Cruz Biotechnology, Santa Cruz, CA, USA.

${ }^{13}$ SC-50384, Santa Cruz Biotechnology, Santa Cruz, CA, USA.

${ }^{14}$ SC-166475, Santa Cruz Biotechnology, Santa Cruz, CA, USA

${ }^{15}$ Axiovision 4.8.2, Carl Zeiss MicroImaging GmbH, Jena, Germany.
}

intra-examiner error, the same sample was analyzed again one week after the first measurement. For morphometric analysis, examiner calibration was assessed by Intraclass Correlation Coefficient (ICC > 0.8). Weighted Cohen's Kappa was used to determine the examiner calibration in the immunohistochemical analyses (>90\%).

\subsection{Statistical analysis of the data}

Changes in the means of the genic expression of IL-1 $\beta$ were defined as the primary outcome variable of the study. The other parameters analyzed were considered secondary outcome variables. Data were grouped and presented as means and standard deviations (continuous variables) or medians, interquartiles ranges and maximum and minimum values (ordinal variables). Normality of the data was verified. The significance of differences among groups in immunohistochemical analysis was determined by Kruskal-Wallis tests, followed by Dunn multiple comparison post hoc test. The data from the other analyses were assessed by analysis of variance (ANOVA) followed by post-hoc Tukey test. The significance level was set at 5\% in all tests.

\section{Results}

\subsection{Clinical follow-up}

All animals tolerated the experimental procedures well and remained healthy all through the experimental period. No significant differences in relation to body weight modification were observed among groups (ANOVA, $\mathrm{p}>0.05$ ).

\subsection{Morphometric analysis}

Animals of groups EP $(1.07 \mathrm{~mm} \pm 0.13)$ and EP-TIL $(0.67 \mathrm{~mm} \pm$ $0.19)$ presented averages of the distance ABC-CEJ significantly greater than the animals of group C $(0.24 \mathrm{~mm} \pm 0.04$; $p<0.05)$. Group EPTIL presented a significant decrease in the average of the distance ABCCEJ when compared with group EP $(\mathrm{p}<0.05)$.

In order to evaluate possible effects of TIL on healthy periodontal tissues, the morphometric analysis was also performed in animals without ligature and receiving TIL (group C-TIL). These animals were administered TIL using the same protocol previously described (1 mg/ $\mathrm{kg}$ body weight TIL solutions injected into the subperiosteal palatal area on days 1, 3, 5, 7 and 9). It was observed that group C-TIL $(0.22 \mathrm{~mm} \pm 0.04)$ presented average of the distance ABC-CEJ not different from group C ( $\mathrm{p}>0.05$; Fig. 1).

\section{3. $q P C R$ analysis}

The qPCR analysis revealed that group EP presented a significant increase in the genic expression levels of IL-1 $\beta$ ( $3.58 \pm 0.42$ fold), TNF- $\alpha$ (13.15 \pm 0.46 fold), MMP-8 (8,766.94 \pm 2.04 fold) and COX-2 (1.49 \pm 0.06 fold) in relation to group C (IL-1 $\beta: 1.02 \pm 0.03$, TNF- $\alpha$ : 


\section{Morphometric Analysis}

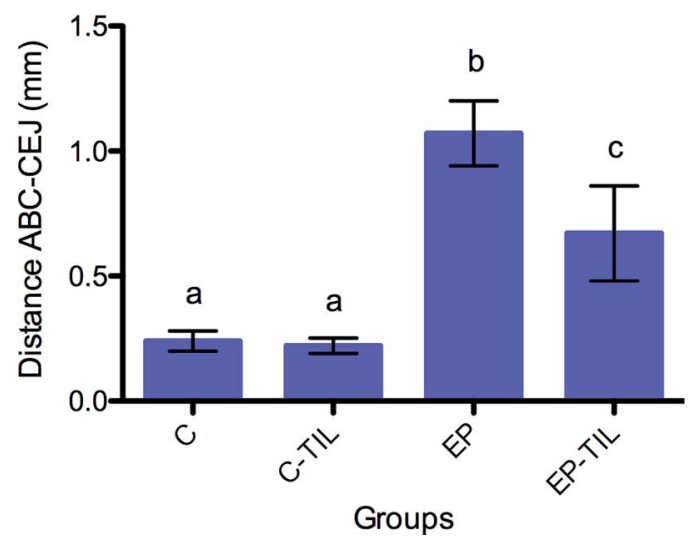

Fig. 1. Morphometric analysis. Means and standard deviations of the linear distance ABCCEJ of the specimens, with comparisons among groups. Same letters indicate no significant differences among groups ( $\mathrm{p}<0.05$; ANOVA, Tukey test).

Abbreviations: $\mathrm{ABC}=$ alveolar bone crest; $\mathrm{CEJ}=$ cementoenamel junction.

$1.09 \pm 0.11$, MMP-8: $1.01 \pm 0.04$ and COX-2: $1.01 \pm 0.03$ fold; $\mathrm{p}<0.05)$. On the other hand, it was observed that the expression of these genes was down-regulated in group EP-TIL (IL-1 $\beta: 0.15 \pm 0.03$, TNF- $\alpha$ : $0.56 \pm 0.06$, MMP-8: $0.01 \pm 0.01$ and COX-2: $0.36 \pm 0.04$ fold) when compared with group EP ( $\mathrm{p}<0.05$; Fig. 2).

\subsection{Immunohistochemical analyses}

The immunohistochemical technique used for detecting IL-1 $\beta$, TNF$\alpha$, MMP-8 and COX-2 presented elevated specificity, as evidenced by the complete absence of labeling in the negative controls for the immunohistochemical reactions. The results of immunohistochemical analyses and photomicrographs showing immunolabeling are presented in Figs. 3-5.

For IL- $1 \beta$, TNF- $\alpha$, MMP- 8 and COX-2, the score 1 prevailed in group $\mathrm{C}$, the score 3 in group EP and the score 2 in group EP-TIL (Fig. 3). The immunolabeling pattern for IL-1 $\beta$, TNF- $\alpha$ and MMP- 8 was reduced in group EP-TIL when compared with group EP ( $\mathrm{p}<0.05$; Figs. 3A-C, 4G-R, 5G-H, K, M-N, Q). Group EP presented higher immunolabeling patterns for all mediators in relation to group $\mathrm{C}(\mathrm{p}<0.05$; Figs. 3, 4AL, 5A-L). Group EP-TIL presented immunolabeling pattern for COX-2 not different from groups C or EP ( $p>0.05$; Figs. 3D, 5C-D, F, I-J, L, O-P, R).

\section{Discussion}

Local administration of BPs allows for the use of higher doses, since they penetrate directly into bone without entering the bloodstream, which favors the achievement of effective concentrations at periodontal tissues (Hikita, Miyazawa, Tabuchi, Kimura, \& Goto, 2009; Reddy et al., 2005). It is expected that local administration of BPs achieve an emphasized and distinctive effect (Hikita et al., 2009). In fact, the accumulation of high levels of BPs in the bone tissue could potentially make them available to the surrounding tissues (Nakaya et al., 2000). In this context, our group performed the first study (Furlaneto et al., 2014) evaluating the effects of TIL on periodontitis. It was concluded that locally administered TIL solutions reduced alveolar bone loss and that the dosage of TIL may influence its anti-inflammatory and antiresorptive properties in EP in rats. Nevertheless, the immuno-inflammatory mechanisms by which TIL might have decreased bone loss and the amount of osteoclasts in this experimental model had not been investigated. Therefore, the present study aimed to evaluate the effects of local administration of TIL on the expression of IL-1 $\beta$, TNF- $\alpha$, MMP-8 and COX-2 in rats with ligature-induced periodontitis.
The placement of ligatures in molars of rats has been extensively used to induce periodontitis and is one of the most representative experimental models of the disease (de Molon et al., 2014). The initiation of periodontitis by bacteria is well-documented in this model, and the destruction of alveolar bone and other connective tissues is readily observed (Lohinai et al., 2001). In this study, the model of periodontitis induction was effective, since group EP presented greater bone loss and up-regulation of important pro-inflammatory mediators involved in periodontal tissue destruction, when compared with group C.

The methodology of the morphometric analysis performed in this study was an adaptation of other method described (de Medeiros Vanderlei et al., 2013). Since periodontitis presents a site-specific nature, the choice of the area to be evaluated may influence the quantification of the alveolar bone loss (Furlaneto et al., 2014). Therefore, we performed measurements of the ABC-CEJ distance in different points of the area that received the ligature. The average of these measurements was used in order to better estimate the dimension of the bone resorption occurred. The results of this study corroborate many others that demonstrated a reduced alveolar bone loss when BPs were administered in EP (Furlaneto et al., 2014; Goya, Paez, \& Mandalunis, 2006; Menezes et al., 2005).

In vitro studies had already shown the inhibition of some pro-inflammatory mediators by TIL (Mönkkönen et al., 1998; Nakaya et al., 2000; Tokuda et al., 1998). However, to our knowledge, this is the first study to demonstrate, in vivo, that TIL is capable of decreasing the genic expression and the immunolabeling of some important mediators involved in periodontal tissue destruction. IL-1 (IL-1 $\alpha$ and IL-1 $\beta$ ) is one of the most potent mediators in osteoclastic bone resorption (Boch, Wara-Aswapati, \& Auron, 2001). Elevated levels of IL-1 $\beta$ have been associated with periodontitis activity and progression (Reinhardt et al., 2010), and reduced levels have been found after periodontal therapy (Sánchez, Miozza, Delgado, \& Busch, 2013). Furthermore, IL-1ß presents synergistic activity with other potent molecules, such as TNF- $\alpha$, in enhancing bone resorption (Stashenko, Dewhirst, Peros, Kent, \& Ago, 1987). It was demonstrated that the BP clodronate alone or combined with low-dose doxycycline significantly decreased the levels of IL-1 $\beta$ expression in ligature-induced periodontitis in rats (Ozdemir et al., 2012). In the present study, TIL reduced the genic expression and immunolabeling of IL-1 $\beta$ in the same experimental model. In fact, it has been shown in vitro that TIL dose-dependently inhibited IL- $1 \beta$ secretion from macrophages (Mönkkönen et al., 1998).

Other actions attributed to IL-1 $\beta$ are proinflammatory effects on osteoblastic cells (Boch et al., 2001; Stashenko et al., 1987) including stimulation of prostaglandin formation and modulation of genic expression of several proteins, including MMPs (Sakaki et al., 2004). MMPs play important roles in tissue-destruction mechanisms associated with periodontitis (Vardar-Sengul et al., 2008); therefore, the inhibition of MMPs by BPs may be considered part of the therapy to prevent tissue destruction in inflammatory diseases (Ozdemir et al., 2012). It has been proposed that BPs may chelate cations $\left(\mathrm{Ca}^{2+}\right.$ and $\left.\mathrm{Zn}^{2+}\right)$ from the MMPs (Teronen et al., 1999), which are completely inactive in the absence of $\mathrm{Ca}^{2+}$ (Okada et al., 1986). MMP-8 is one of the most important collagenases in the extracellular matrix degradation in periodontal tissues (Vardar-Sengul et al., 2008). It can contribute to the disease ethiopathogenesis also through inactivation of proteinase inhibitors and through non-matrix substrates including chemokines that are neutrophil chemoattracts (Sorsa et al., 2006). It was evidenced that inflamed rat tissues can in vivo express MMP-8 mRNA and protein (Pirilä et al., 2001), which was also observed in the present study. In fact, it was demonstrated that some BPs, combined or not with other drugs, can significantly inhibit MMP-8 expression in samples from sites presenting periodontitis and peri-implantitis (Buduneli et al., 2007; Teronen et al., 1997). It is known that TIL is capable of restraining the activity of both MMP-1 and MMP-3 in human periodontal ligament cells (Nakaya et al., 2000), which is a significant finding since the inhibition of MMP activity at the bone surface may block an initiating step in the 


\section{qPCR Analyses}
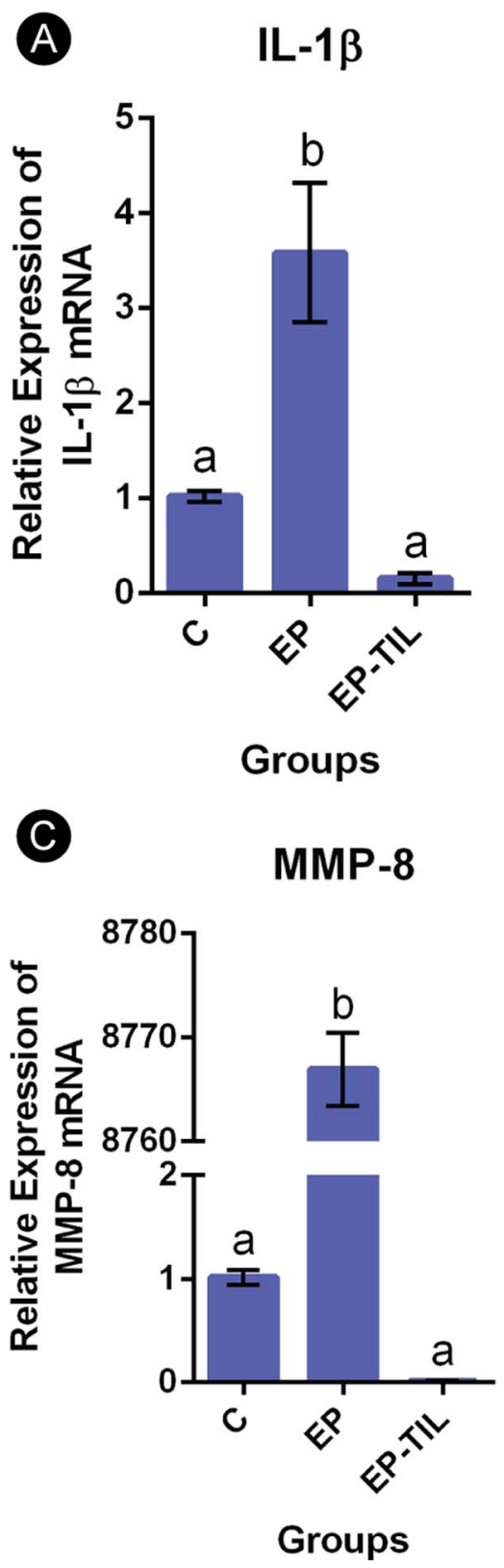

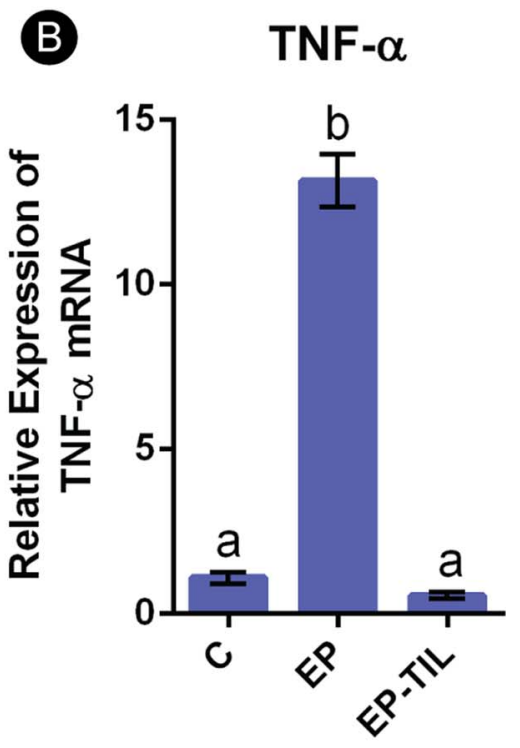

Groups

(D)

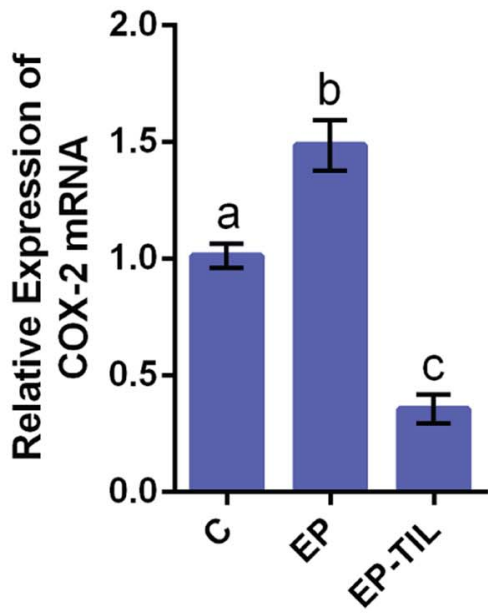

Groups
Fig. 2. Quantitative reverse transcription-polymerase chain reaction analyses. Means and standard deviations of the relative genic expression of IL$1 \beta$ (A), TNF- $\alpha$ (B), MMP-8 (C) and COX-2 (D), with comparison among groups. Same letters indicate no significant differences between groups (p < 0.05; ANOVA,Tukey test). bone resorption process (McCauley \& Nohutcu, 2002). Nevertheless, to our knowledge, the effects of TIL on MMP- 8 expression had not been evaluated yet. Our results demonstrated that this BP can inhibit MMP-8 genic expression and immunolabeling in vivo.

TNF- $\alpha$ is an early proinflammatory cytokine in the periodontal inflammatory process and is known to induce osteoclastic activity, viability, and differentiation and, therefore, stimulate bone resorption (Graves \& Cochran, 2003). This cytokine also contributes to periodontitis through other mechanisms, such as the induction of adhesion molecules and other mediators that facilitate the inflammatory response and the stimulation of MMP expression and apoptosis of matrixproducing cells (Graves \& Cochran, 2003). It has been demonstrated that BPs can exert their anti-inflammatory properties by decreasing the levels of TNF- $\alpha$ and IL-1 $\beta$ (Bianchi, Franchi, Ferrario, Sotgiu, \& Sacerdote, 2008). The capacity of TIL to inhibit TNF- $\alpha$ secretion was demonstrated in vitro (Mönkkönen et al., 1998). The present study showed that TIL is also capable of reducing the TNF- $\alpha$ genic expression and immunolabeling in vivo.

COX-2 was also investigated in the present study, since it plays an important role in periodontitis by mediating inflammatory reactions in periodontal tissues (Schaefer et al., 2010). COX-2 and its products may regulate the production of some MMPs, such as MMP-8 (Bezerra et al., 2000). It was also reported that IL-1 $\beta$ stimulates MMP-1 expression in human gingival fibroblasts, and the expression may be mediated through the induction of COX-2 and $\mathrm{PGE}_{2}$ generation (Sakaki et al., 2004). In fact, selective COX-2 inhibitors are capable of reducing alveolar bone loss in EP in rats (Bezerra et al., 2000; Holzhausen, Rossa Júnior, Nassar, Spolidório, \& Spolidório, 2002). It has been demonstrated that non-nitrogen-containing BPs, such as clodronate, etidronate and TRK-530, inhibit COX-2 expression in vitro (Liu et al., 2006; Shinoda, Takeyama, Suzuki, Murakami, \& Yamada, 2008; Suzuki et al., 2007). On the other hand, TIL failed to modulate the expression of COX2 in cultured monocytes (Tuominen et al., 2006). The results of the present study contradict the latter, since we demonstrated the potential 


\section{Immunohistochemical Analyses}

A

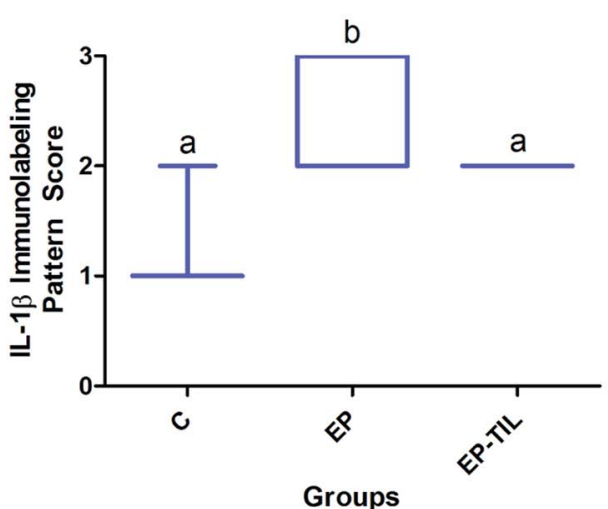

C

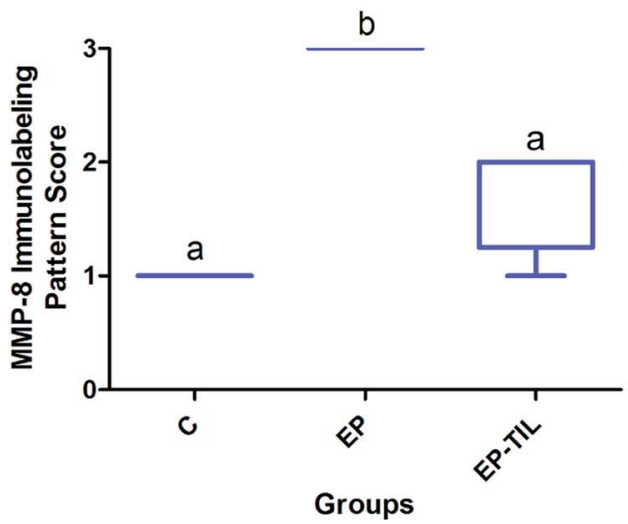

\section{IL-1 $\beta$}

B

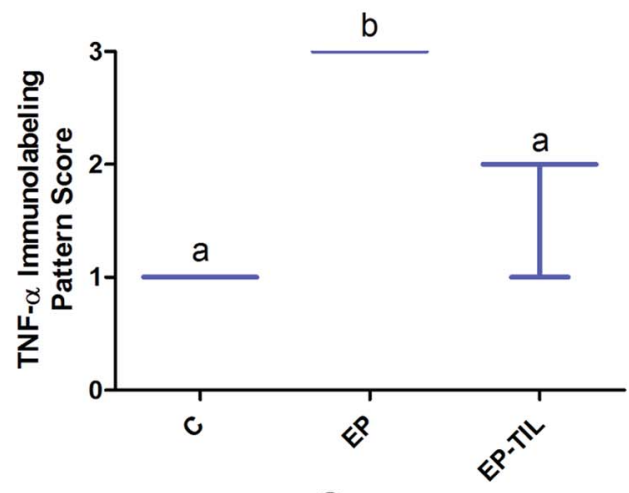

Groups

D

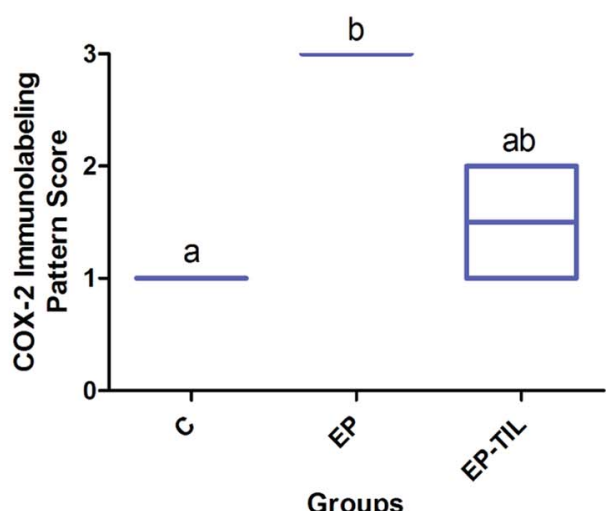

Fig. 3. Immunohistochemical analyses. Medians, interquartile ranges, maximum and minimum values of the immunolabeling scores for IL-1 $\beta$ (A), TNF- $\alpha$ (B), MMP-8 (C) and COX-2 (D). Same letters indicate no significant differences between groups $(\mathrm{p}<0.05$; Kruskal-Wallis, Dunn tests). of TIL to reduce COX-2 genic expression in EP in rats. The immunolabeling of COX-2, however, was not different from the control and EP groups. In point of fact, although genic expression assays are usually in accordance with local protein expression tests, discrepancies might be found (Oda et al., 2010), thus showing that the amount of mRNA of certain molecule is not necessarily correlated to its tissue expression (Abdul Murad et al., 2013).

The present study demonstrated reduced expressions of important mediators involved in periodontal tissue destruction due to local administrations of TIL in periodontal tissues. It is important to notice, however, that immunomodulatory therapies, such as the one used in this research, do not totally disrupt the inflammatory response, instead, they allow it to happen in a more balanced manner (Gulati, Anand, Govila, \& Jain, 2014). Besides the anti-resorptive and anti-inflammatory effects of TIL on EP, demonstrated in this study and previously (Furlaneto et al., 2014), other advantage of this BP is that it has a prolonged skeletal-retention time, leading to a continual biologic effect (Delmas, Vergnaud, Arlot, Pastoureau, \& Meunier, 1991). Also, TIL is a harmless compound and presents a considerable therapeutic margin because it can effectively inhibit bone resorption without reducing bone mineralization and strength (Bonjour, Ammann, Barbier, Caverzasio, \& Rizzoli, 1995). It is important to emphasize that, since TIL is a non-nitrogen-containing BP, the likelihood of occurrence of side effects associated with nitrogen-containing BPs, such as osteonecrosis of the jaw
(Diel et al., 2007), is minimized. In the present study, TIL was administered in healthy periodontal tissues also, without any side effect. In fact, the group without ligature receiving TIL presented the same features as the animals of the control group. Therefore, there is a potential for a clinical use of local administrations of TIL in the management of periodontal diseases.

It is important to emphasize that this research presents the inherent limitations of an experimental study in rats. Other investigations should be performed to study the range of the effective local concentration of TIL in periodontal tissues, the ideal frequency and dosage of administration as well as the duration of the effect before a clinical application could be proposed. Further preclinical studies are also important to clarify the possible participation of other inflammatory mediators through which TIL influences periodontal tissues. Clinical trials are needed to investigate if the treatment with TIL would improve the results of the conventional periodontal therapy currently used (mechanical control of the dental biofilm), especially in patients more susceptible to periodontitis, with impaired tissue healing and immunoinflammatory response.

\section{Conclusion}

Within the limits of this study, it can be concluded that locally administered TIL solutions downregulate important mediators involved in 


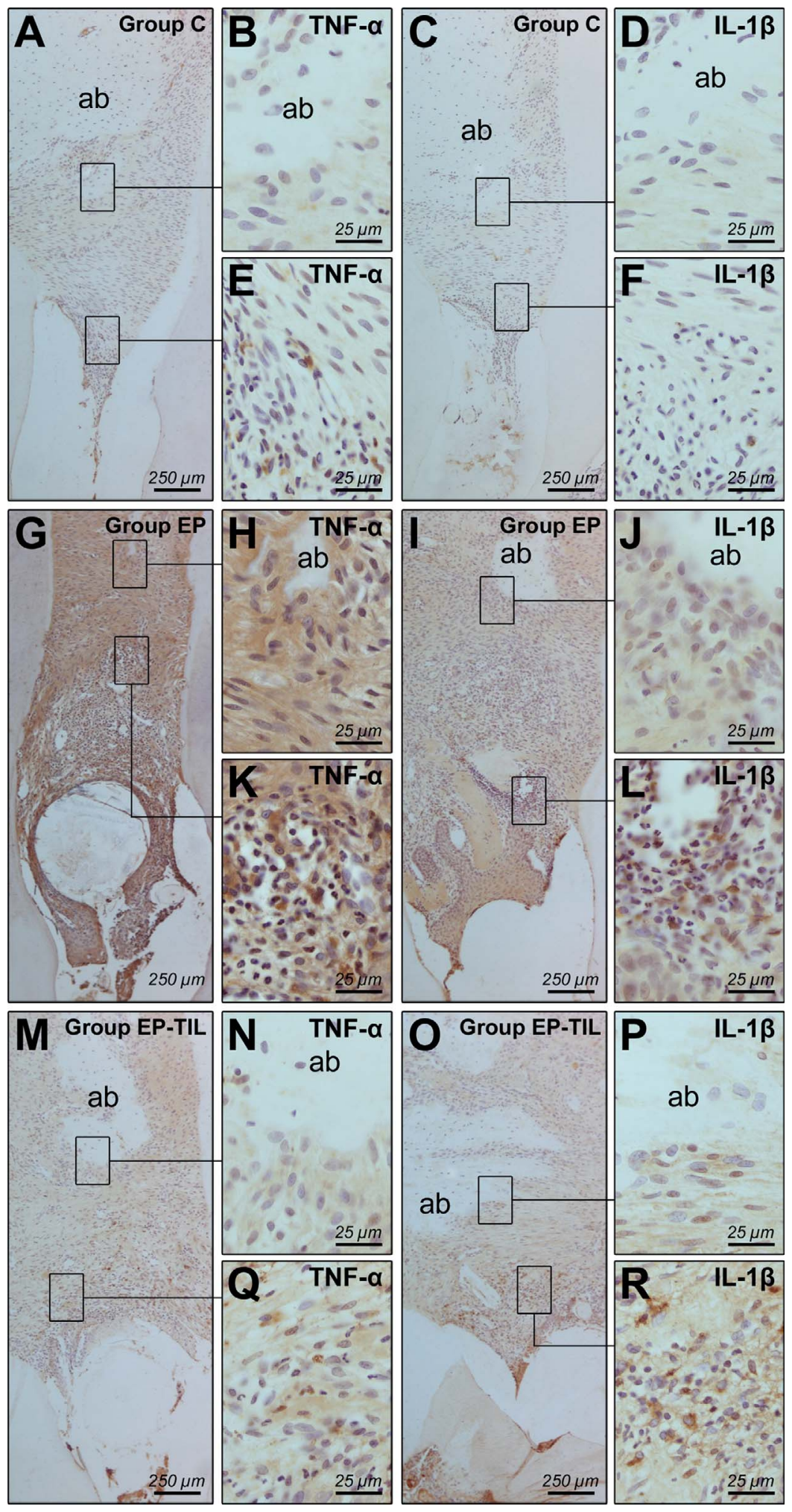

Fig. 4. Photomicrographs showing immunolabeling for TNF- $\alpha$ (A, B, E, G $\mathrm{H}, \mathrm{K}, \mathrm{M}, \mathrm{N}$ and $\mathrm{Q}$ ) and IL-1 $\beta$ (C, D, F, I, J, L, O, P and R) in the interproximal area between the 1st and 2nd molars in groups C (A-F), EP (G-L) and EP-TIL (M-R).

Abbreviations: $a b=$ alveolar bone. Magnification: $A C, G, I, M$ and $\mathrm{O}$ : x100; BD, E, F, H, J, K, L, N, P, Q and R: x1000. Scale bars: A, C, G, I, M and O: $250 \mu \mathrm{m}$; BD, E, F, H, J, K, L, N, P, Q and R: $25 \mu \mathrm{m}$ (Hematoxylin counterstaining).

\section{Funding}

This work was supported by the Foundation for Support in Scientific and Technological Development of Ceara (FUNCAP, Fortaleza, CE, Brazil) and Coordination for the Improvement of Higher Education Personnel (CAPES, Brasilia, DF, Brazil) - Grant 23038.009502/2013

\section{Conflict of interest}

There is no relationship between any author and commercial firms that may pose a conflict of interest. 


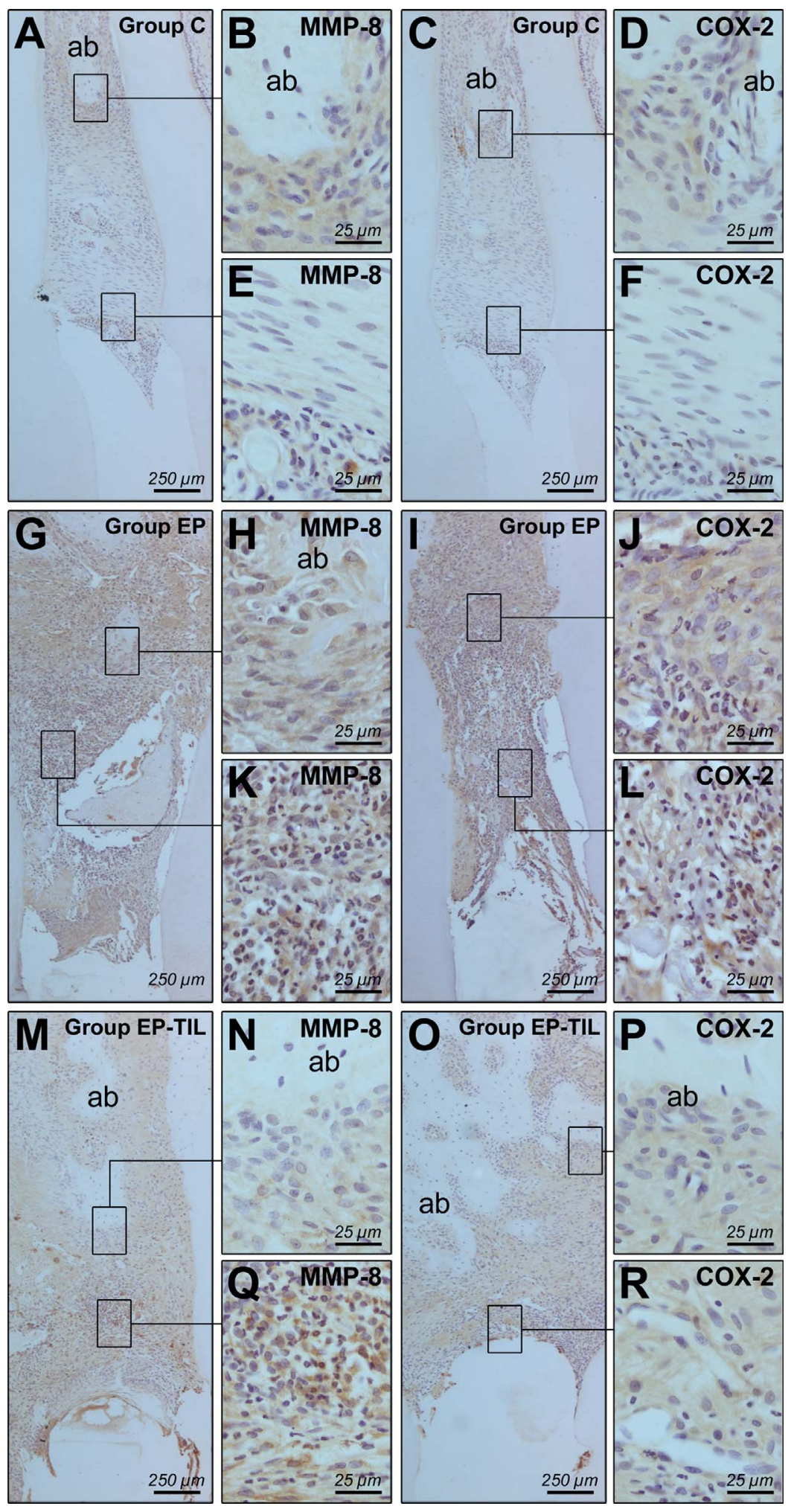

Fig. 5. Photomicrographs showing immunolabeling for MMP-8 (A, B, E, G, $\mathrm{H}, \mathrm{K}, \mathrm{M}, \mathrm{N}$ and $\mathrm{Q}$ ) and COX-2 (C, D, F, I, J, L, O, P and R) in the interproximal area between the 1 st and 2nd molars in groups C (A-F), EP (G-L) and EP-TIL (M-R). Abbreviations: $a b=$ alveolar bone. Magnification: A, C, G, I, M and O: x100; B, D, E, F, H, J, K, L, N, P, Q and R: x1000. Scale bars: A, C, G, I, M and O: $250 \mu \mathrm{m}$; B, D, E, F, H, J, K, L, N, P, Q and R: $25 \mu \mathrm{m}$ (Hematoxylin counterstaining).

\section{Acknowledgement}

The authors thank Adalberto Lima Junior (Laboratory Assistant, Federal University of Ceará - UFC, Brazil) for his support during the preparation of the histologic specimens.

\section{References}

Abdul Murad, N. A., Razak, Z. A., Hussain, R. M., Syed Hussain, S. N., Ko Ching Huat, C., Che Md Ali, S. A., ... Jamal, R. (2013). Quantification of Her-2/Neu gene in breast cancer patients using real time-polymerase chain reaction (Q-PCR) and correlation with immunohistochemistry findings. Asian Pacific Journal of Cancer Prevention, 14, 1655-1659.

Bezerra, M. M., de Lima, V., Alencar, V. B., Vieira, I. B., Brito, G. A., Ribeiro, R. A., \& Rocha, F. A. (2000). Selective cyclooxygenase-2 inhibition prevents alveolar bone loss in experimental periodontitis in rats. Journal of Periodontology, 71, 1009-1014. 
Bianchi, M., Franchi, S., Ferrario, P., Sotgiu, M. L., \& Sacerdote, P. (2008). Effects of the bisphosphonate ibandronate on hyperalgesia, substance $\mathrm{P}$, and cytokine levels in a rat model of persistent inflammatory pain. European Journal of Pain, 12, 284-292.

Boch, J. A., Wara-Aswapati, N., \& Auron, P. E. (2001). Interleukin 1 signal transductioncurrent concepts and relevance to periodontitis. Journal of Dental Research, 80, 400-407.

Bonjour, J. P., Ammann, P., Barbier, A., Caverzasio, J., \& Rizzoli, R. (1995). Tiludronate: Bone pharmacology and safety. Bone, 17, 473S-477S.

Buduneli, E., Vardar-Sengul, S., Buduneli, N., Atilla, G., Wahlgren, J., \& Sorsa, T. (2007). Matrix metalloproteinases, tissue inhibitor of matrix metalloproteinase-1, and laminin-5 gamma2 chain immunolocalization in gingival tissue of endotoxin-induced periodontitis in rats: Effects of low-dose doxycycline and alendronate. Journal of Periodontology, 78, 127-134.

Coura, C. O., Souza, R. B., Rodrigues, J. A., Vanderlei Ede, S., de Araújo, I. W., Ribeiro, N. A., ... Benevides, N. M. (2015). Mechanisms involved in the anti-inflammatory action of a polysulfated fraction from Gracilaria cornea in rats. PLoS One, 10, e0119319. http://dx.doi.org/10.1371/journal.pone.0119319.

D'Aoust, P., McCulloch, C. A., Tenenbaum, H. C., \& Lekic, P. C. (2000). Etidronate (HEBP) promotes osteoblast differentiation and wound closure in rat calvaria. Cell and Tissue Research, 302, 353-363.

David, P., Nguyen, H., Barbier, A., \& Baron, R. (1991). The bisphosphonate tiludronate is a potent inhibitor of the osteoclast vacuolar $\mathrm{H}(+)$-ATPase. Journal of Bone and Mineral Research, 11, 1498-1507.

de Medeiros Vanderlei, J. M., Messora, M. R., Fernandes, P. G., Novaes, A. B., Jr., Palioto, D. B., de Moraes Grisi, M. F., ... Taba, M., Jr. (2013). Arterial hypertension perpetuates alveolar bone loss. Clinical and Experimental Hypertension, 35, 1-5.

de Molon, R. S., de Avila, E. D., Boas Nogueira, A. V., Chaves de Souza, J. A., AvilaCampos, M. J., de Andrade, C. R., \& Cirelli, J. A. (2014). Evaluation of the host response in various models of induced periodontal disease in mice. Journal of Periodontology, 85, 465-477.

Delmas, P. D., Vergnaud, P., Arlot, M., Pastoureau, P., \& Meunier, P. (1991). The in viva anabolic effect of hPTH(1-34) is blunted when bone resorption is blocked by a bisphosphonate. Journal of Bone and Mineral Research, 6, S136.

Diel, I. J., Fogelman, I., Al-Nawas, B., Hoffmeister, B., Migliorati, C., Gligorov, J., .. Aapro, M. S. (2007). Pathophysiology, risk factors and management of bisphosphonate-associated osteonecrosis of the jaw: Is there a diverse relationship of aminoand non-aminobisphosphonates? Critical Reviews in Oncology/Hematology, 64, 198-207.

Furlaneto, F. A., Nunes, N. L., Oliveira Filho, I. L., Frota, N. P., Yamamoto, K. O., Lisboa, M. R., ... Messora, M. R. (2014). Effects of locally-administered Tiludronic Acid on experimental periodontitis in rats. Journal of Periodontology, 85, 1291-1301.

Goya, J. A., Paez, H. A., \& Mandalunis, P. M. (2006). Effect of topical administration of monosodium olpadronate on experimental periodontitis in rats. Journal of Periodontology, 77, 1-6.

Graves, D. T., \& Cochran, D. (2003). The contribution of interleukin-1 and tumor necrosis factor to periodontal tissue destruction. Journal of Periodontology, 74, 391-401.

Guenther, H. L., Guenther, H. E., \& Fleisch, H. (1981). The effects of 1-hydroxyethane-1,1diphosphonate and dichloromethanediphosphonate on collagen synthesis by rabbit articular chondrocytes and rat bone cells. Biochemical Journal, 196, 293-301.

Gulati, M., Anand, V., Govila, V., \& Jain, N. (2014). Host modulation therapy: An in dispensable part of perioceutics. Journal of Indian Society of Periodontology, 18, $282-288$.

Hikita, H., Miyazawa, K., Tabuchi, M., Kimura, M., \& Goto, S. (2009). Bisphosphonate administration prior to tooth extraction delays initial healing of the extraction socket in rats. Journal of Bone and Mineral Metabolism, 27, 663-672.

Holzhausen, M., Rossa Júnior, C., Marcantonio, E., Jr., Nassar, P. O., Spolidório, D. M., \& Spolidório, L. C. (2002). Effect of selective cyclooxygenase-2 inhibition on the development of ligature-induced periodontitis in rats. Journal of Periodontology, 73 , 1030-1036.

Kirkwood, K. L., Cirelli, J. A., Rogers, J. E., \& Giannobile, W. V. (2007). Novel host response therapeutic approaches to treat periodontal diseases. Periodontology, 43(2000), 294-315.

Lane, N., Armitage, G. C., Loomer, P., Hsieh, S., Majumdar, S., Wang, H. Y., ... Munoz, T. (2005). Bisphosphonate therapy improves the outcome of conventional periodontal treatment: Results of a 12-month, randomized, placebo-controlled study. Journal of Periodontology, 76, 1113-1122.

Lisboa, M. R., Gondim, D. V., Ervolino, E., Vale, M. L., Frota, N. P., Nunes, N. L., Furlaneto, F. A. (2015). Effects of electroacupuncture on experimental periodontitis in rats. Journal of Periodontology, 86, 801-811.

Liu, L., Igarashi, K., Kanzaki, H., Chiba, M., Shinoda, H., \& Mitani, H. (2006). Clodronate inhibits PGE(2) production in compressed periodontal ligament cells. Journal of Dental Research, 85, 757-760.

Livak, K. J., \& Schmittgen, T. D. (2001). Analysis of relative gene expression data using real-time quantitative PCR and the 2(-Delta Delta C(T)) Method. Methods, 25, 402-408.

Lohinai, Z., Stachlewitz, R., Székely, A. D., Fehér, E., Dézsi, L., \& Szabó, C. (2001) Evidence for the expression of cyclooxygenase-2 enzyme in periodontitis. Life Science, 70, 279-290.

Mönkkönen, J., Simila, J., \& Rogers, M. J. (1998). Effects of tiludronate and ibandronate on the secretion of proinflammatory cytokines and nitric oxide from macrophages in vitro. Life Science, 62, PL95-102.

McCauley, L. K., \& Nohutcu, R. M. (2002). Mediators of periodontal osseous destruction and remodeling: Principles and implications for diagnosis and therapy. Journal of Periodontology, 73, 1377-1391.

Menezes, A. M., Rocha, F. A., Chaves, H. V., Carvalho, C. B., Ribeiro, R. A., \& Brito, G. A.
(2005). Effect of sodium alendronate on alveolar bone resorption in experimental periodontitis in rats. Journal of Periodontology, 76, 1901-1909.

Murakami, H., Takahashi, N., Tanaka, S., Nakamura, I., Udagawa, N., Nakajo, S., ... Suda, T. (1997). Tiludronate inhibits protein tyrosine phosphatase activity in osteoclasts. Bone, 20, 399-404.

Nakaya, H., Osawa, G., Iwasaki, N., Cochran, D. L., Kamoi, K., \& Oates, T. W. (2000). Effects of bisphosphonate on matrix metalloproteinase enzymes in human periodontal ligament cells. Journal of Periodontology, 71, 1158-1166.

Oda, M., Arihiro, K., Kataoka, T., Osaki, A., Asahara, T., \& Ohdan, H. (2010). Comparison of immunohistochemistry assays and real-time reverse transcription-polymerase chain reaction for analyzing hormone receptor status in human breast carcinoma. Pathology International, 60, 305-315.

Okada, Y., Nagase, H., \& Harris, E. D., Jr. (1986). A metalloproteinase from human rheumatoid synovial fibroblasts that digests connective tissue matrix components. Purification and characterization. The Journal of Biological Chemistry, 261, $14245-14255$.

Ozdemir, S. P., Kurtiș, B., Tüter, G., Bozkurt Ș, Gültekin, S. E., Sengüiven, B., ... Aydın, S. (2012). Effects of low-dose doxycycline and bisphosphonate clodronate on alveolar bone loss and gingival levels of matrix metalloproteinase- 9 and interleukin-1s in rats with diabetes: A histomorphometric and immunohistochemical study. Journal of Periodontology, 83, 1172-1182.

Pirilä, E., Ramamurthy, N., Maisi, P., McClain, S., Kucine, A., Wahlgren, J., ... Sorsa, T. (2001). Wound healing in ovariectomized rats: Effects of chemically modified tetracycline (CMT-8) and estrogen on matrix metalloproteinases $-8,-13$ and type I collagen expression. Current Medicinal Chemistry, 8, 281-294.

Reddy, G. T., Kumar, T. M., \& Veena (2005). Formulation and evaluation of Alendronate Sodium gel for the treatment of bone resorptive lesions in Periodontitis. Drug Delivery, $12,217-222$

Reinhardt, R. A., Stoner, J. A., Golub, L. M., Lee, H. M., Nummikoski, P. V., Sorsa, T., \& Payne, J. B. (2010). Association of gingival crevicular fluid biomarkers during periodontal maintenance with subsequent progressive periodontitis. Journal of Periodontology, 81, 251-259.

Rogers, M. J., Frith, J. C., Luckman, S. P., Coxon, F. P., Benford, H. L., Mönkkönen, J., .. Russell, R. G. (1999). Molecular mechanisms of action of bisphosphonates. Bone, 24, 73S-79S.

Russell, R. G., Xia, Z., Dunford, J. E., Oppermann, U., Kwaasi, A., Hulley, P. A., ... Ebetino, F. H. (2007). Bisphosphonates: An update on mechanisms of action and how these relate to clinical efficacy. Annals of the New York Academy of Sciences, 1117, 209-257.

Sánchez, G. A., Miozza, V. A., Delgado, A., \& Busch, L. (2013). Salivary IL-1ß and PGE2 as biomarkers of periodontal status, before and after periodontal treatment. Journal of Clinical Periodontology, 40, 1112-1117.

Sakaki, H., Matsumiya, T., Kusumi, A., Imaizumi, T., Satoh, H., Yoshida, H., ... Kimura, H. (2004). Interleukin-1beta induces matrix metalloproteinase-1 expression in cultured human gingival fibroblasts: Role of cyclooxygenase-2 and prostaglandin E2. Oral Diseases, 10, 87-93.

Schaefer, A. S., Richter, G. M., Nothnagel, M., Laine, M. L., Noack, B., Glas, J., .. Schreiber, S. (2010). COX-2 is associated with periodontitis in Europeans. Journal of Dental Research, 89, 384-388.

Sharma, A., \& Pradeep, A. R. (2012). Clinical efficacy of $1 \%$ alendronate gel as a local drug delivery system in the treatment of chronic periodontitis: A randomized, controlled clinical trial. Journal of Periodontology, 83, 11-18.

Shinoda, H., Takeyama, S., Suzuki, K., Murakami, S., \& Yamada, S. (2008). Pharmacological topics of bone metabolism: A novel bisphosphonate for the treat ment of periodontitis. Journal of Pharmacological Sciences, 106, 555-558.

Silverman, S. L. (2008). Paget disease of bone: Therapeutic options. Journal of Clinical Rheumatology, 14, 299-305.

Sorsa, T., Tjäderhane, L., Konttinen, Y. T., Lauhio, A., Salo, T., Lee, H. M., ... Mäntylä, P. (2006). Matrix metalloproteinases: Contribution to pathogenesis: Diagnosis and treatment of periodontal inflammation. Annals of Medicine, 38, 306-321.

Stashenko, P., Dewhirst, F. E., Peros, W. J., Kent, R. L., \& Ago, J. M. (1987). Synergistic interations between interleukin 1, tumor necrosis fator and lymphotoxin in boné resorption. The Journal of Immunology, 138, 1464-1468.

Suzuki, Y., Nishiyama, T., Hasuda, K., Fujishiro, T., Niikura, T., Hayashi, S., ... Kurosaka, M. (2007). Effect of etidronate on COX-2 expression and PGE(2) production in macrophage-like RAW 264.7 cells stimulated by titanium particles. Journal of Orthopaedic Science, 12, 568-577.

Teronen, O., Konttinen, Y. T., Lindqvist, C., Salo, T., Ingman, T., Lauhio, A., ... Sorsa, T. (1997). Human neutrophil collagenase MMP-8 in peri-implant sulcus fluid and its inhibition by clodronate. Journal of Dental Research, 76, 1529-1537.

Teronen, O., Heikkilä, P., Konttinen, Y. T., Laitinen, M., Salo, T., Hanemaaijer, R., ... Sorsa, T. (1999). MMP inhibition and downregulation by bisphosphonates. Annals of the New York Academy of Sciences, 878, 453-465.

Tokuda, H., Kozawa, O., Harada, A., \& Uematsu, T. (1998). Tiludronate inhibits interleukin-6 synthesis in osteoblasts: Inhibition of phospholipase D activation in MC3T3E1 cells. Journal of Cell Biochemistry, 69, 252-259.

Tuominen, O. M., Ylitalo-Heikkala, R., Vehmas, T. I., Mucha, I., Ylitalo, P., \& Riutta, A. (2006). Effects of bisphosphonates on prostaglandin E2 and thromboxane B2 production in human whole blood and monocytes stimulated by lipopolysaccharide and A23187. Methods and Findings in Experimental and Clinical Pharmacology, 28, 361-367.

Vardar-Sengul, S., Buduneli, E., Turkoglu, O., Buduneli, N., Atilla, G., Wahlgren, J., ... Baylas, H. (2008). The effects of selective COX-2 inhibitor/Celecoxib and omega-3 fatty acid on matrix metalloproteinases, TIMP-1, and laminin-5g2-chain immunolocalization in experimental periodontitis. Journal of Periodontology, 79 , 1934-1941. 